
.

\title{
Two phase behaviour and the distribution of volume
}

\author{
VASILIKI PLEROU, PARAMESWARAN GOPIKRISHNAN $\dagger$ and \\ H. EUGENE STANLEY*
}

\author{
Center for Polymer Studies and Department of Physics, Boston University, \\ Boston, Massachusetts 02215, USA
}

(Received 17 August 2005; in final form 11 October 2005)

The short term dynamics of stock prices is largely driven by fluctuations in demand and supply. A balance between demand and supply for the stock results only in mild price fluctuations; in contrast, a large imbalance can cause considerable price movement. Using transactions and quotes data for 116 most-actively traded US stocks for the 2 yr period 1994-1995, we showed empirically that the probability distribution of demand $\Omega$ conditioned on its local noise intensity $\Sigma$ displays a change from a unimodal distribution for $\Sigma<\Sigma_{c}$, to a bi-modal distribution for $\Sigma>\Sigma_{c}$ (Plerou et al. 2003).

We hypothesized that these empirical findings can be interpreted as indicating that, as $\Sigma$ changes, the stock market moves between an 'equilibrium' state during which the price fluctuates around its consensus value, and an 'out-of-equilibrium' state during which there is an excess of buyers or sellers and the stock is evolving to a new consensus value. Lastly, we remarked on the analogy of this bifurcation phenomenon to similar results in phase transition phenomena, and interpreted the different states as distinct 'phases'.

Following the procedure of Plerou et al. (2000), we define demand $\Omega=\sum q_{i} a_{i}$, where $q_{i}$ is the trade size of trade $i, a_{i}$ indicates buy $\left(a_{i}=1\right)$ or sell $\left(a_{i}=-1\right)$ and the

*Corresponding author. Email: hes@bu.edu

$\uparrow$ Present address: Goldman Sachs \& Co., New York.

$\$$ A similar bifurcation is found also by conditioning on $Q$. summation is over the $N$ trades in $\Delta t$. In a recent article, Matia and Yamazaki (2005) (MY) construct $\Omega$ in a similar way from transactions and quotes data for 102 stocks during a different time period 2001-2002. They then construct $P(\Omega \mid \Sigma)$ and confirm our empirical finding of a change of this distribution from an unimodal to a bimodal behaviour as $\Sigma$ crosses a threshold $\Sigma_{c}$.

MY then perform a numerical simulation where $\Omega$ and $\Sigma$ are constructed from $q$ and $N$ with the previouslyreported power-law exponents $\zeta_{N}=3.4$ (Plerou et al. 2000) and $\zeta_{q}=3 / 2$ (Gopikrishnan et al. 2000). From this randomly generated time series, $\mathrm{MY}$ find that the distribution $P(\Omega \mid \Sigma)$ changes from an unimodal to a bimodal shape as $\Sigma$ increases. They further observe that the bifurcation behaviour is absent when $P(q)$ is replaced by a Gaussian distribution. MY conclude that the empirically-observed 'two phase behaviour' is a consequence of the fat-tailed distribution of volume. A similar explanation was previously proposed by Potters and Bouchaud (2003).

In our simulation, we construct time series for $\Omega$ and $\Sigma$ using the same number of data points as in our empirical data, corresponding to 116 stocks each with length 12896 data points. Figure 1 shows $P(\Omega \mid \Sigma)$ for $\Omega$ constructed from a simulation using (a) power-law distributed $q$ with exponent $\zeta_{q}=3 / 2$, (b) power-law distributed $q$ with exponent $\zeta_{q}=3$ and (c) exponentially distributed $q$. Figure 1(a) shows that the bifurcation behaviour is clearly evident for the case $\zeta_{q}=1.5$, as found by MY. Figures $1(b)$ and $(c)$ show that a similar bifurcation is 

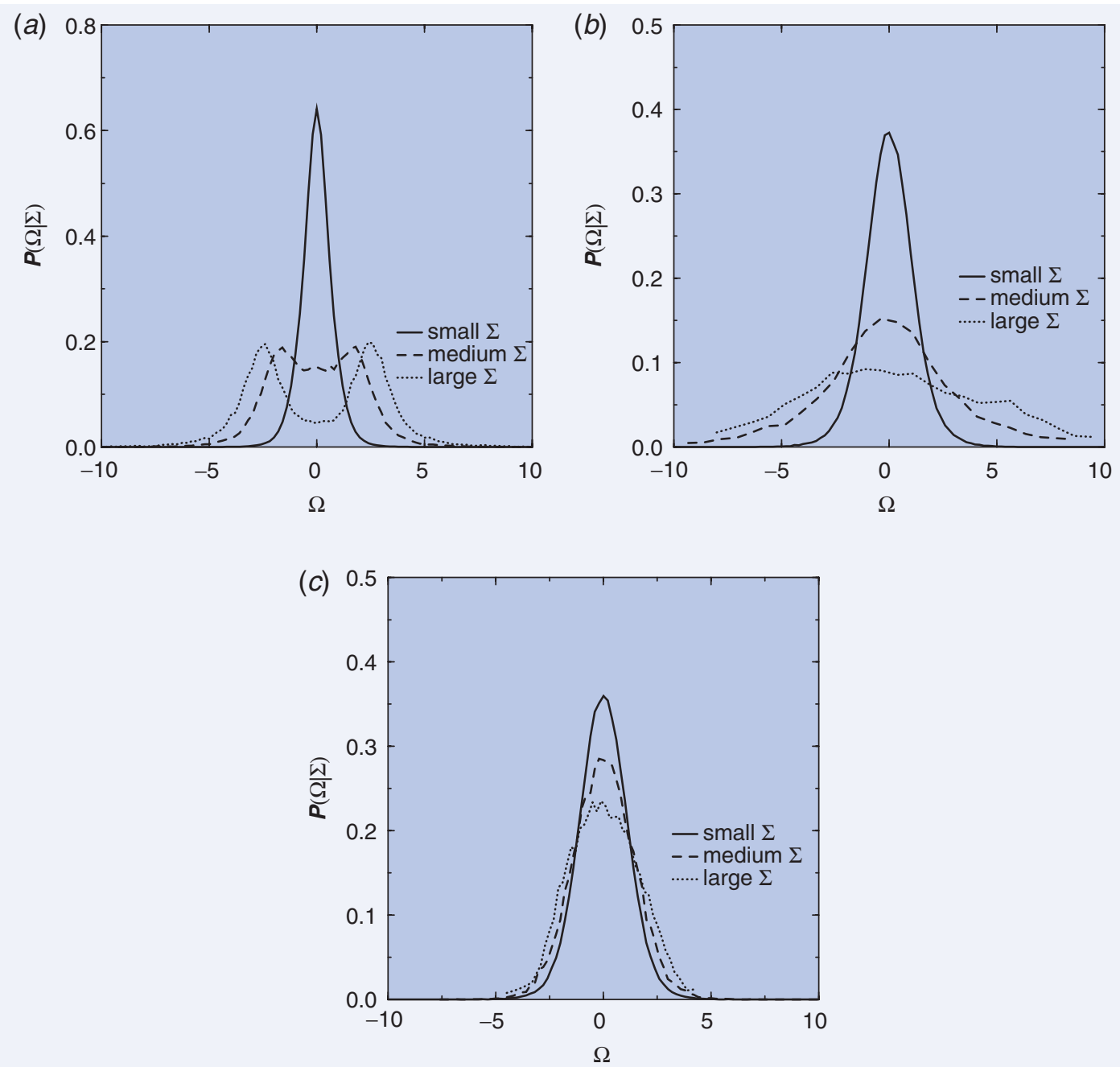

Figure 1. Conditional distribution $P(\Omega \mid \Sigma)$ from generated time series with $(a)$ power-law distribution of $q$ with exponent $\zeta_{q}=3 / 2$, (b) power-law distribution of $q$ with exponent $\zeta_{q}=3$ and (c) exponential distribution of $q$. We show three bins of $\Sigma$, the first corresponds to a small value (solid line) and the last (dotted line) corresponds to the bin with the largest value of $\Sigma$ in our sample. Here the generated time series have a length identical to the length of our time series (116 stocks for 2 years sampled at 15 min intervals). We have in all three cases chosen $N$ from a power-law distribution with $\zeta_{N}=3.4$ calibrated for each time series to have the same mean value as the empirical data for each stock, and the random sign $a_{i}$ is taken from $[-1,+1]$. Here $\Omega$ is set to zero mean and normalized by the first centred moment and $\Sigma$ is normalized by its first moment.

not evident for $\zeta_{q}=3$ and for exponentially distributed $q$ respectively. $\dagger$

For Lévy stable distributed $P(q)$, as is the case for trade sizes, a large $\Sigma$ (or large $Q$ ) is often due to a few large trades, so when the volume is large, the demand is likely to have the direction of the largest trade. In other words, when there is large volume, demand is (statistically) directional (either buy or sell). The effect of $\zeta_{q}<2$ is very significant here; in contrast, returns (tail exponent $\left.\zeta_{G}>2\right)$ under conditions of high volatility measured from intra-day data for example, do not acquire a statistically significant direction.

Our results are in agreement with the conclusion of MY that the change of $P(\Omega \mid \Sigma)$ from an unimodal to a bimodal distribution (Plerou et al. 2003) is an artifact of the distribution of trade sizes $q$ which are power-law distributed with exponent $\zeta_{q}<2$ in the Lévy stable domain (Potters and Bouchaud 2003). Although the bifurcation behaviour is a consequence of the distribution of trade sizes, the corresponding market states have a real

$\dagger$ Increasing the time series length significantly, one finds a similar bifurcation for the exponential and the $\zeta_{q}=3$ cases as well, although for the time series length and the average $N$ in our empirical data, this effect is not discernible. In the limit of large (constant) $N$, only the cases $\zeta_{q}<2$ will show the bifurcation, since others will converge to a Gaussian distribution of $\Omega$. 


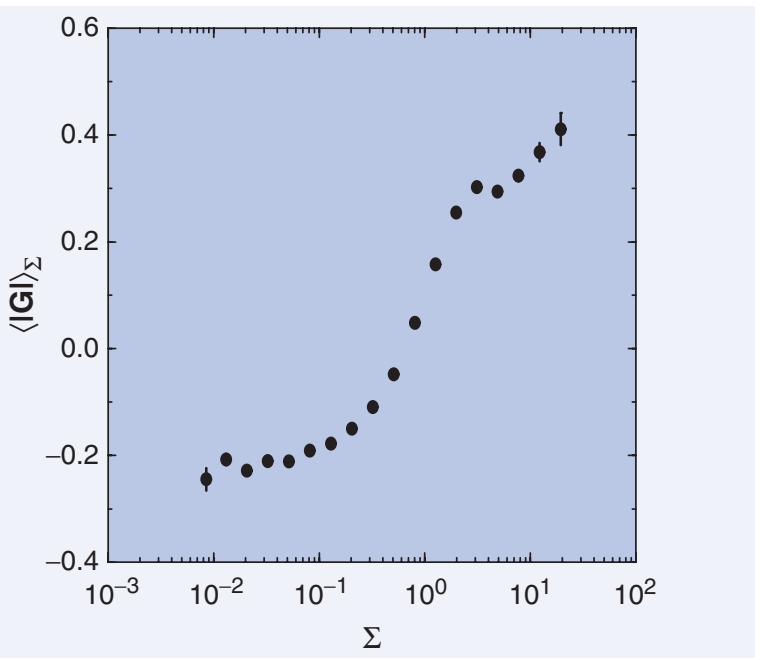

Figure 2. Conditional expectation of the absolute value of the returns $\langle|G|\rangle_{\Sigma}$, as a function of $\Sigma$ for the 116 most actively traded stocks in our sample for the 2 yr period 1994-1995. For each stock, $|G|$ is set to zero mean (so, the negative value of the ordinate) and normalized by its standard deviation, and $\Sigma$ is normalized by its first centred moment.

significance and interpretation. Under regular market conditions when the volume traded is comparable to the market depth (small $Q$ or $\Sigma$ ), prices may tend to fluctuate mildly around their local equilibrium values, while for volumes much larger than the depth (equivalently for large $\Sigma$ ), prices move more significantly. For example, figure 2 shows the conditional expectation $\mathbf{E}(|G| \mid \Sigma)$ for different $\Sigma$. We find that for small $\Sigma, \mathbf{E}(|G| \mid \Sigma)$ has small values, so prices fluctuate mildly, while $\mathbf{E}(|G| \mid \Sigma)$ for large $\Sigma$ shows larger values corresponding to large price movements.

\section{Acknowledgments}

We thank J.-P. Bouchaud and M. Potters for helpful interactions on this topic and X. Gabaix for helpful comments on this draft.

\section{References}

Gopikrishnan, P., Plerou, V., Gabaix, X. and Stanley, H.E., Phys. Rev. E, 2000, 62, 4493.

Matia, K. and Yamazaki, K., Statistical properties of demand fluctuation in the financial market. Quant. Finance, 2005, 5, 513-517

Plerou, V., et al., Phys. Rev. E, 2000, 62, R3023.

Plerou, V., et al., Nature, 2003, 421, 130.

Potters, M. and Bouchaud, J.-P., Comment on two phase behavior of financial markets, eprint: cond-mat/0304514, 2003. 